\title{
Maintenance of Muscle Contraction Improves during Dynamic Exercise by Multi-channel Functional Electrical Stimulation with Time Shifting Stimulation
}

\author{
Rintaro Kamihira ${ }^{1 *}$, Misato Kasuya ${ }^{1}$, Yinlai Jiang ${ }^{1,2}$, Shunta Togo ${ }^{1}$, Masao Sugi ${ }^{1}$, Hiroshi Yokoi ${ }^{1,2}$ \\ ${ }^{1}$ Graduate School of Informatics and Engineering, the University of Electro-Communications, Tokyo, Japan. \\ 2 Brain Science Inspired Life Support Research Center, the University of Electro-Communications, Tokyo, \\ Japan. \\ * Corresponding author. Tel.: +81-042-443-5403; email: yokoi@mce.uec.ac.jp \\ Manuscript submitted July 12, 2016; accepted December 26, 2016. \\ doi: 10.17706/ijbbb.2017.7.1.33-40
}

\begin{abstract}
Functional electrical stimulation (FES) is an effective tool for rehabilitation. However the decrease of induced muscle contraction is one of the limitations of using FES in clinical therapy. The purpose of this study is to improve the evoked tension by periodical shifting of stimulating pair of electrode on the same muscle. We applied the time shifting stimulation (TSS) to biceps brachii to validate its effect. Two pairs of surface electrodes were placed over biceps brachii's motor point. Motor point moves depending on muscle contraction, so the electrode pairs were set to stimulate the motor point in elbow flexion posture and extension posture respectively. TSS changed the stimulating pair of electrode periodically from the distal pair to the proximal pair with 0.5 seconds offset. For comparison, simultaneous stimulation (SS) that stimulated two pairs of electrodes at the same time was conducted. The improvement of maintenance of muscle contraction was assessed by the angle of motion induced by FES. Ten healthy male subjects performed in both TSS and SS experiments in which 180 contractions were induced by FES in 15 minutes. 1 week rest was taken between the stimulation methods. As a result, TSS realized significantly larger angle of motion than SS. The effect of TSS on angle of motion maintained for 15 minutes. The results suggest that TSS may cause less fatigue than SS. The effectiveness of TSS can be contributed by stimulating motor point periodically. Because motor point moves from distal position to proximal position in response to the muscle contraction, TSS may apply stimulation to motor point effectively.
\end{abstract}

Key words: Functional electrical stimulation, muscle fatigue, biceps brachii, motor point, multi-channel electrode.

\section{Introduction}

Functional Electrical Stimulation (FES) is an effective therapeutic tool for patients who suffer from strokes or spinal cord injuries [1]. FES is used as a rehabilitation method that induces muscle contractions to help restore motor function. FES can assist limb motion with applying alternating current stimulation. Non-invasive FES provides stimulation from surface electrode placed on the skin, so it is relatively easy to apply to a clinical therapy for safety. In order to use FES for a dynamic exercise such as walking and cycling, multi-channel FES are used as an effective rehabilitation tool for support the more difficult rehabilitation task [2]. Multi-channel FES can activate different coordination group of muscles so patient can train various motions involving multi degree of freedom. 
However, one of the biggest limitations of non-invasive FES is that it rapid decreases the muscle contraction and weakens muscle response [3]. The limitation can decrease the angular torque or angle of motion induced by the stimulation. The decrease of the maintenance of muscle contraction leads to a difficulty of continuing rehabilitation for enough time and the restoration of motor function may delay. Various methods have been studied to avoid reducing the strength of muscle contraction [4], [5]. It is suggested that when stimulation is applied to a motor point, less current is required to elicit the muscle contraction. A motor point locates where motor branch of a nerve enters muscle belly [6]. The electrical stimulation to motor point excites motor axon so that all muscle fibers connected to the motor axon contract. Currently Nguyen et al., have shown that a method using multichannel FES called Spatially Distributed Sequential Stimulation (SDSS) is effective in keeping muscle torque [4]. SDSS has three or four active electrodes which are placed on different muscle groups and one reference electrode. The stimulation electrode is shifted periodically among these three or four active electrodes in order to prolong the muscle contraction of each muscle group. These studies applied FES to quadriceps [7] or triceps surae [4] that several muscle groups share a common function like knee extension or planter flexion. However there are many muscle groups that only one muscle group is involved in a limb motion such as elbow extension or flexion or ankle dorsiflexion. SDSS is difficult to apply to such muscle groups, which limits the applications of SDSS.

Our objective is to develop a method that can improve the resistance of reducing the contraction during elbow flexion. Only one muscle, biceps brachii, has a motor point [8] contributes to elbow flexion.

Our method is the periodic shifting of active and reference electrodes from the distal part of the biceps to the proximal part. This method is called time shifting stimulation (TSS) (see Fig. 1). To investigate the effect of the TSS, we compare it with another method called simultaneous stimulation (SS) (see Fig. 1) in which stimulation from two channels applied simultaneously.

Various studies on the reduction of muscle contraction by FES assessed muscle response with angular torque. However in present study, we investigated the influence on the maintenance of muscle contraction with angle of motion because in clinical rehabilitation, passive range of motion is one of the important indexes to assess the motor recovery [9].
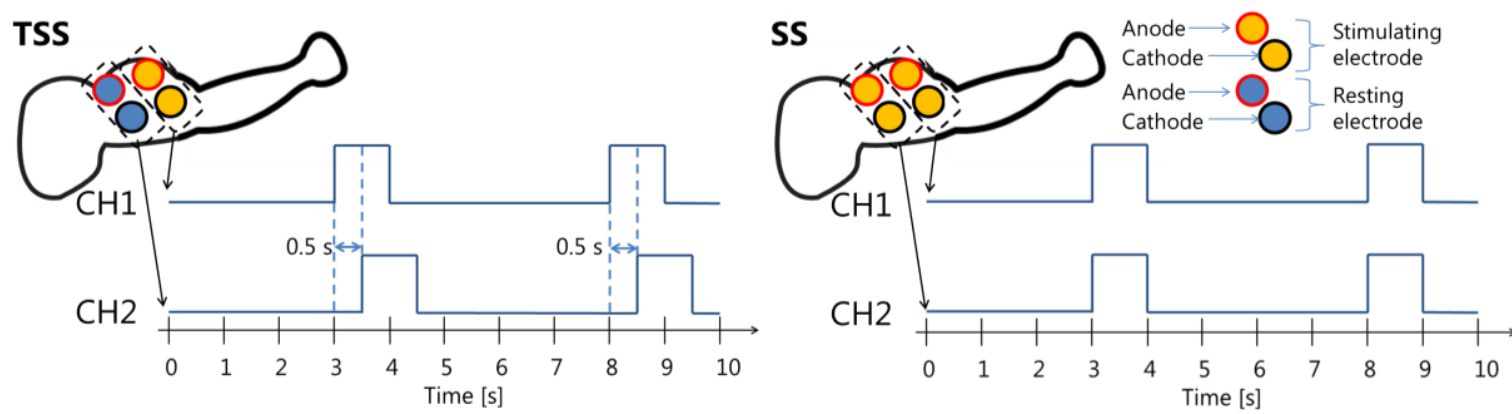

Fig. 1. Schematic representation of TSS and SS.

Electrode pairs were placed vertical to the muscle fiber. Stimulation timing of each channel is shown below the electrode placement. Stimulation in channel 2 is delayed 0.5 second in TSS. Stimulation timing in both channels of SS is same. Stimulation duration is 1 second. The wave shape of stimulation voltage is shown in Fig. 2.

\section{Methods and Materials}

\subsection{Subjects}


10 healthy male subjects in their 20's were recruited. The study was approved by the ethical committee at The University of Electro-Communications. Each subject signed a written informed consent before the experiment.

\subsection{Device}

Elbow angle ( $\theta$ in Fig. 3) was measured using an electro-goniometer with a sampling frequency of $10 \mathrm{~Hz}$ (Model SG150 twin-axis goniometer; Biometric Ltd., Ladysmith, VA). A programmable 4-ch constant voltage FES stimulator (System Instruments Co., Ltd.) was used to apply electrical stimulation. Stimulation parameters and ON/OFF control signal of stimulator would send from PC1 (see Fig. 3). FES stimulation and measurement of elbow angle were synchronized.

\subsection{Stimulation}

Self-adhesive circular electrodes (radius: $5 \mathrm{~cm}$ ) were placed over the motor point of the biceps. Stimulation was alternating current with $2000 \mathrm{~Hz}$ carrier frequency (pulse width: $500 \mu \mathrm{s}$ ) modulated at $100 \mathrm{~Hz}$ (duty cycle: 50\%). The burst modulated alternating current stimulation was suggested to induce muscle strength with less discomfort [10]. Stimulation intensity was adjusted based on the angle of motion generated by FES. Stimulation intensity was set to the minimum voltage which was able to rotate elbow angle more than 30 degrees of motion from the initial position.

\subsection{Experimental Settings}

Before the experiment, the positions of the motor point were searched for with a pen electrode [4] when the arm was flexed and extended. Two pairs of stimulation electrodes were placed over the two positions.

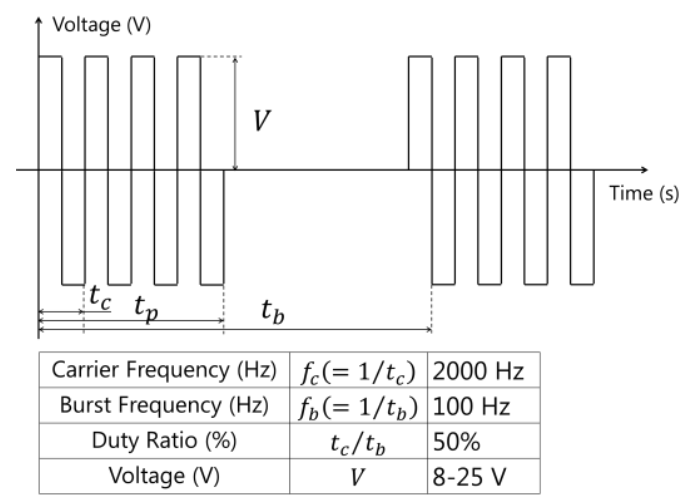

Fig. 2. Stimulation shape in TSS and SS.

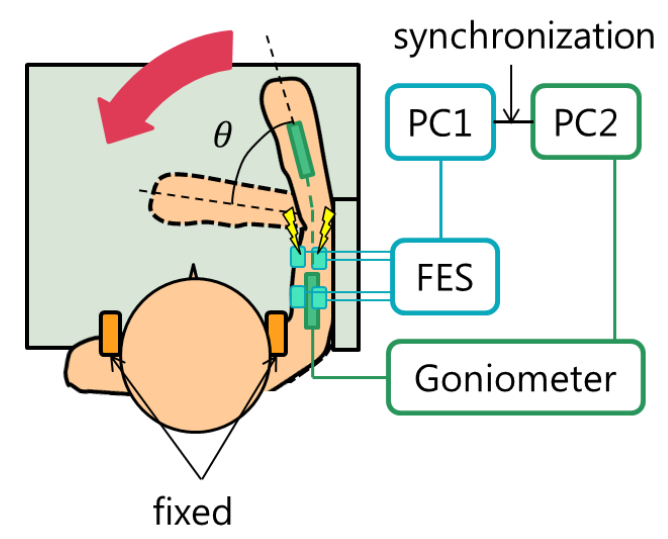

Fig. 3. Schematic diagram of the experimental environment. 


\subsection{Task}

Each subject made his/her arm perform elbow flexion by FES on the transverse plane (range of motion: 98.2-71.3 (degree)) and returned his/her arm to full extension position voluntarily in one set (see Fig. 3). One trial consisted of continuous 180 sets of exercise (15 minutes). In the first trial SS was applied to the subject and in the second trial, TSS was applied. The first trial and second trial was separated by 1 week to allow the subjects to have enough rest.

\subsection{Data Analysis}

The mean peak angle of motion of certain length of data (180 contractions or 18 contractions) was averaged across the 10 subjects. a paired t-test was performed. The statistical significance was accepted if $p \leq 0.05$. Data depicted as a bar graph with mean \pm standard deviation.

\section{Result}

Fig. 4 (a) shows the time series data for the elbow angle of a representative subject. The elbow angle induced by SS continued to go up and down but the elbow angle induced by TSS constantly maintained at a high level for 15 minutes.

The angle positions in the first 90 contractions are shown in Fig. 4 (b). Each point indicates the peak angle of motion in one contraction. In the first 90 seconds, difference between the maximum angle of motion and minimum angle of motion was 39.2 degree at SS and 8.8 degree at TSS. The reduction of angle of motion of TSS was much less than that of SS.
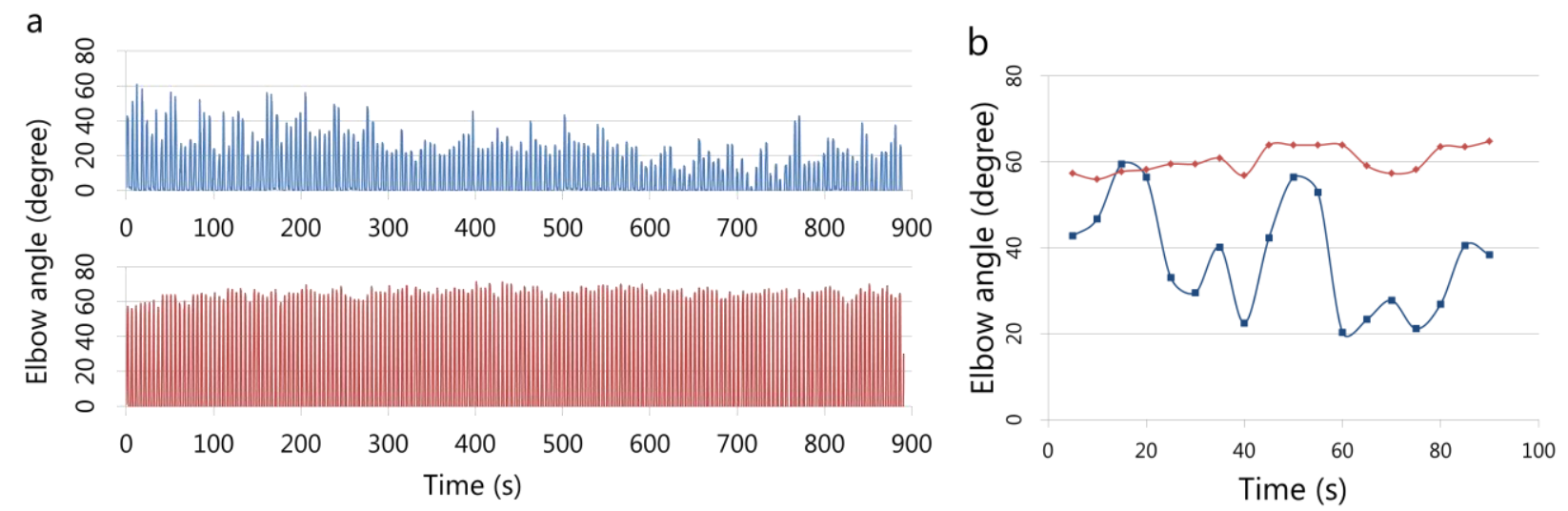

Fig. 4. Elbow angle of one representative subject (Blue: SS, Red: TSS). (a) Time series data of peak angle of motion induced by FES with 180 contractions (15 minutes). Blue: SS, Red: TSS. (b) Peak elbow angle of the representative subject in first 18 contractions (90 seconds). Each point is shown peak angle of motion in one contraction.

Fig. 5 (a) shows the mean peak angular position for 15 minutes averaged across 10 participants. Paired $t$-test was used on the average angle of motion data of the 10 subjects to compare the effect of SS and TSS on maintaining angular position. According to Fig. 5 (a), TSS induced a wider range of elbow angle than SS significantly $(p \leq 0.05)$.

Although most subjects' time series data of angle of motion in SS was shown a tendency similar to the representative subject shown in Fig. 4, there were three subjects whose time series data of angle of motion in SS was relatively stable. Time series data of TSS was also stable in the two subjects whose time series data in SS was stable. Only one subject out of ten had an opposite effect of TSS that resulted in less angle of motion than SS. 
Fig. 5(b) and Fig. 5(c) show the average peak elbow angle of contractions in the first and the last 90 seconds. The average elbow angle with TSS in both the first and the last 90 seconds is significantly larger than with SS. Thus it is shown that the effect of TSS maintains significantly.

These results suggest TSS is more effective in maintaining larger angle of motion induced by FES than SS.

a

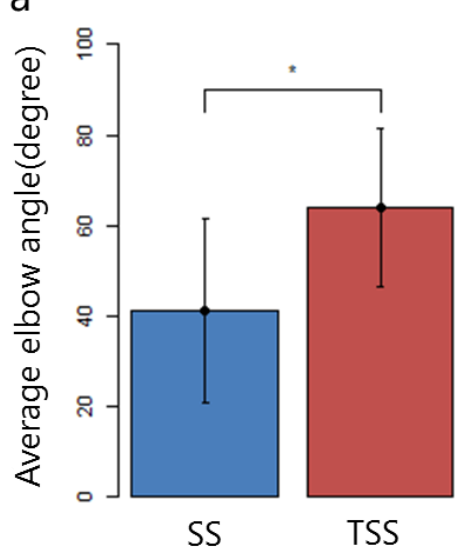

b

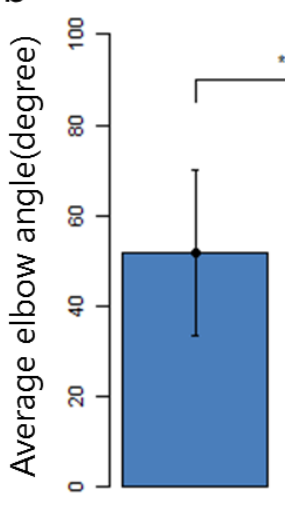

SS

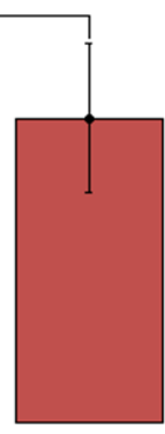

TSS

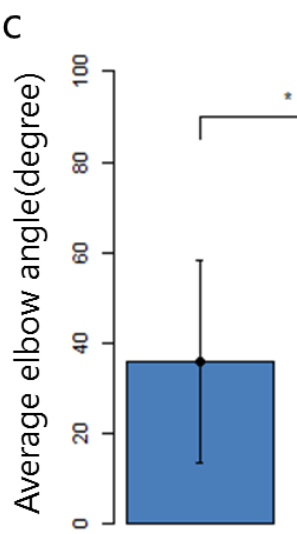

SS

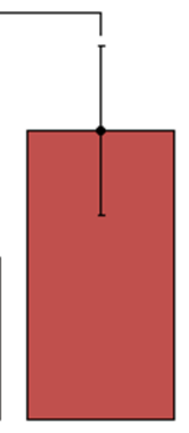

TSS

Fig. 5. Average elbow angle of ten subjects ( $p \leq 0.05$ ) (Blue: SS, Red: TSS). (a) The Mean angle of motion of 180 contractions (15 minutes) was averaged across the 10 subjects. (b) Mean angle of motion of first 18 contractions (90 seconds) was averaged across ten subjects. (c) Mean angle of motion of last 18 contractions (90 seconds) was averaged across ten subjects.

\section{Discussion}

In this study, the effect of TSS on keeping muscle contraction was demonstrated by a wide angle of motion in 10 healthy subjects.

The two pairs of electrode were placed over the motor point position when arm was extended and flexed. Stimulation was shifted from the distal pair of electrodes to the proximal pair based on the fact that elbow muscle contraction makes the motor point to move from distal position to proximal position during elbow flexion. We assume that TSS may stimulate over the motor point effectively. According to the study, we hypothesize that time shifting of the stimulation electrode in response to motor point movement may be effective in maintaining muscle contraction. However the improvement of the maintenance of angle of motion was not consistent in all the subjects. We developed FES system in which pairs of stimulation electrodes can be shifted with limb motion. The muscle length and limb angle show a linear correlation [11]. The movement of motor point can be tracked with feedback from angular position. As a future work we will develop closed-loop FES system with angle feedback and improve the effect of the maintenance of angle of motion.

The opposite effect of TSS in only one subject out of ten may occur when the two pairs of electrodes were so close that the output current from both pairs of electrode is overlapped. In the previous study [4], the effect of SDSS was shown despite an overlap of stimulation. However the length of pair of electrodes becomes much closer in applying stimulation to biceps. In this case, the size of electrode for TSS needs to be optimized in case of current overlapping.

In this study, the effect of TSS was assessed by the results of ten able-bodied subjects. TSS may also be applicable in rehabilitation of paralyzed patients whose fatigue resistance weaker than healthy people [12].

\section{Conclusion}


The effect of TSS in maintaining muscle contraction was validated by an experiment of 10 healthy subjects. TSS can be applied to muscle groups with a single muscle group activation related to one limb motion, to which SDSS may not be applicable. The experimental results suggested that TSS in response to motor point movement could have an influence on maintaining muscle contraction. We are working on developing multi-channel FES which can change the stimulation electrode in response to angular position and will conduct further study as future work.

\section{Acknowledgement}

We thank all of people who contributed to this study and gratefully acknowledge the support by System Instruments Co., Ltd. for providing us with FES device. This research was partially supported by the "Brain Machine Interface Development" from Japan Agency for Medical Research and Development, AMED and JSPS KAKENHI Grant Number JP26120008.

\section{References}

[1] Popovic, M. R., Curt, A., Keller, T., \& Dietz, V. (2001). Functional electrical stimulation for grasping and walking: indications and limitations. Spinal Cord., 403-412.

[2] Qian, J. G., Rong, K., Qian, Z., Wen, C., \& Zhang, S. (2015). Effects of a multichannel dynamic functional electrical stimulation system on hemiplegic gait and muscle forces. Journal of Physical Therapy Science, 27(11), 3541-3544.

[3] Binder-Macleod, S. A., \& Snyder-Mackler, L. (1993) .Muscle fatigue: Clinical implications for fatigue assessment and neuromusclar electrical stimulation. Physical Therapy Journal, 73(12), 902-910.

[4] Nguyen, R., Masani, K., Micera, S., Morari, M., \& Popovic, M. R. (2011). Spatially distributed sequential stimulation reduces fatigue in paralyzed triceps surae muscles: a case study. Artificial Organs, 35(12), 1174-1180.

[5] Thrasher, A., Graham, G. M., \& Popovic, M. R., (2005). Reducing muscle fatigue due to functional electrical stimulation using random modulation of stimulation parameters. Artificial Organs, 29(6), 453-458.

[6] Gobbo, M., Maffiuletti, N. A., Orizio, C., \& Minetto, M. A. (2014). Muscle motor point identification is essential for optimizing neuromuscular electrical stimulation use. Journal of NeuroEngineering and Rehabilitation, 13(76).

[7] Pournezam, M., Andrews, B. J., Baxendale, R. H., Phillips, G. F., \& Paul, J. P., (1988). Reduction of muscle fatigue in man by cyclical stimulation. Journal of Biomedical Engineering, 10(2), 196-200.

[8] John, H. W. (1993) The Extremities and The Head, Neck, and Trunk: Muscle and Motor Points, Lea \& Febiger.

[9] Jong, L. D., Dijkstra, P. U., Stewart, R. E., \& Postema, K. (2012). Repeated measurements of arm joint passive range of motion after stroke: interobserver reliability and sources of variation. Physical Therapy Journal, 92(8), 1027-1035.

[10] Selkowitz, D. M., Rossman, E. G., \& Fitzpatrick, S. (2009). Effect of burst-modulated alternating current carrier frequency on current amplitude required to produce maximally tolerated electrically stimulated quadriceps femoris knee extension torque. American Journal of Physical Medicine \& Rehabilitation, 88(12), 973-978.

[11] Ning, L., \& Xin, H. (2012). Fusimotor control of spindle sensitivity regulates central and peripheral coding of joint angles. Frontiers in Computational Neuroscience, 66(6).

[12] Pelletier, C. A., \& Hicks, A. L. (2011). Muscle fatigue characteristics in paralyzed muscle after spinal cord injury, Spinal Cord, 49(1), 125-130. 


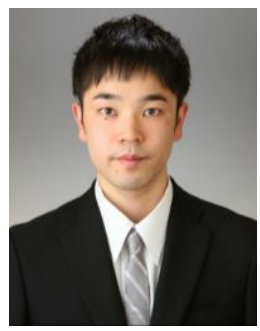

Rintaro Kamihira is a graduate student in the Department of Mechanical Engineering and Intelligent Systems in Graduate School of Informatics and Engineering, the University of Electro-Communications. He received the B.E. from the University of Electro-Communications in 2014.

His current interests are biomedical engineering, muscle fatigue, and multi-channel functional electrical stimulation.

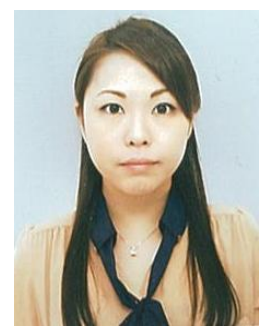

Misato Kasuya is a PhD. student in Graduate School of Informatics and Engineering, the University of Electro-Communications since 2014. She received the B.E., the M.E. from Seikei University in 2009 and 2011, respectively.

Her research subjects are recovery of motor control for paralysis patients by functional electrical stimulation. She presented 3 oral presentations in national and international conference.

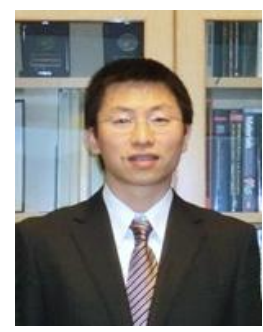

Yinlai Jiang He received a Ph.D in engineering from Kochi University of Technology, Japan, in 2008. He was a research associate from 2008 to 2012, and an assistant professor from 2013 to 2014 in Kochi University of Technology. He is currently an associate professor in the Brain Science Inspired Life Support Research Center, The University of Electro-Communications.

His current research interests biological engineering, robotics, and human robot interface. He is currently a member of IEEE, The Robotic Society of Japan, Japanese Society for Medical and Biological Engineering, and Japan Society for Fuzzy Theory and Intelligent Informatics

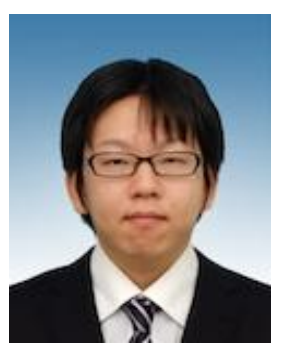

Shunta Togo is an assistant professor in Graduate School of Informatics and Engineering, the University of Electro-Communications since 2016. He received the B.E., the M.E. and the PhD. from Nagoya University in 2009, 2011 and 2014, respectively. He was a Japan Society for the Promotion of Science (JSPS) research fellow (DC2) in Graduate School of Engineering, Nagoya University from 2012 to 2014. He was a JSPS research fellow (PD) in Cognitive Mechanisms Laboratories, Advanced Telecommunications Research Institute International (ATR) from 2012 to 2016.

He teaches undergraduate courses in Advanced Robotics Program in Department of Mechanical Engineering and Intelligent Systems. His current interests are human motor control, motor coordination, and myoelectric control device. In addition, an approach to control mechanism of human multi-joint and multi-muscle is his research subjects. He has published 10 research articles and presented 28 oral or poster presentations in national and international conference.

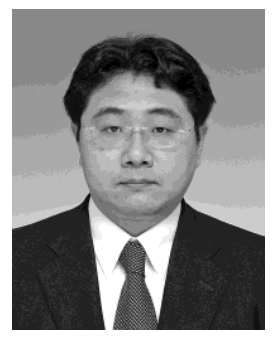

Masao Sugi was born in Tokyo, Japan in 1974. He received the B.E. degree in mathematical engineering in 1998 and the M.E. and Ph.D. degrees in precision engineering in 2000 and 2003, respectively, from the University of Tokyo. His research interests are decentralized autonomous systems, manufacturing systems, and human-robot interaction.

He was a project research associate from 2003 to 2007, a Project Assistant Professor from 2007 to 2009, and a Project Researcher from 2009 to 2010, respectively, at The University of Tokyo. From 2010 to 2011 he was an Assistant Professor at Tokyo University 
of Agriculture and Technology. Since 2011, he has been an Associate Professor at Department of Mechanical Engineering and Intelligent Systems, Graduate School of Informatics and Engineering, The University of Electro-Communications.

Dr. Sugi is a member of the IEEE, the Society of Instrument and Control Engineers, the Robotics Society of Japan, the Japan Society of Mechanical Engineers, the Japan Society for Precision Engineering, and the Society for Serviceology.

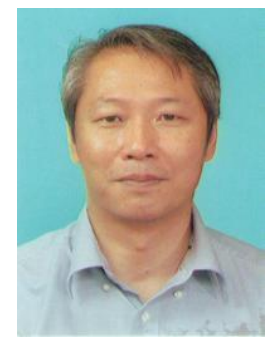

Hiroshi Yokoi was born in Nagoya, Japan, in 1963. He received B.S., M.S., and Ph.D. degrees in precision engineering from Hokkaido University, Japan, in 1986, 1990, and 1993, respectively. He was an engineer at Toyota Motor Cooporation from 1996 to 1997. He joined as the Researcher, Institute of Bioscience and Human Technology, AIST form 1993-1995. He was an associate professor in the Department of Complex System Engineering of Faculty of Engineering at Graduate School of Hokkaido University from 1995 to 2004, and the Department of Precision Engineering, Faculty of Engineering, the University of Tokyo from 2004 to 2009. He is a senior researcher in AI-Lab at the University of Zurich, from 2002 and also a Fellow Researcher in IAS-Lab at the University of West England form 2003, and is working as a guest professor, National Institute of Physiological Science from 2009.

$\mathrm{He}$ is currently professor of the Department of Mechanics and Intelligence at the University of Electro-Communication, and guest professor of Interfaculty Initiative in Information at the University of Tokyo. His current research interests include computational intelligence in robotics, artificial life, medical engineering. 